\title{
Some aspects of Amacrine neuron simulation for motion detection
}

\author{
J.A. Martin-Pereda (jamp@atf.upm.es), A.P. Gonzalez-Marcos (agonmar@itfo.upm.es) \\ E.T.S. Ingenieros de Telecomunicación. Universidad Politécnica de Madrid \\ Ciudad Universitaria. 28040 Madrid. Spain
}

\begin{abstract}
As it is known, there are five types of neurons in the mammalian retinal layer allowing the detection of several important characteristics of the visual image impinging onto the visual system. namely. photoreceptors. horizontal cells. amacrine, bipolar and ganglion cells. And it is a well known fact too, that the amacrine neuron architecture allows a first detection for objects motion, being the most important retinal cell to this function. We have already studied and simulated the Dowling retina model and we have verified that many complex processes in visual detection is performed with the basis of the amacrine cell synaptic connections. This work will show how this structure may be employed for motion detection.
\end{abstract}

Keywords: amacrine, motion detection, logic cells.

\section{INTRODUCTION}

The retina is one of the more complex sensory elements in any living being. It is the basic sense to interact with other animals around and gives the main references for connecting with the outer world. Because these facts, it is able to perform very complex functions, going from motion to shape detection. According to the complexity of the living being, the number of these performed functions can be very high and, in most of the cases, they are much more complex that any of the nowadays achieved with artificial systems.

The mammalian retina is one of the more sophisticated existing systems and, when working together with the visual cortex, gives to mammals one of their more powerful tools to interact with other animals and the world around. It is because that, some parts of its architectural structure may be employed as models to construct artificial visual systems. For example, signals obtained from the retina give information about some types of motion, colors, shapes and velocities of the bodies around. Every one of these characteristics is obtained with just five layers of neurons. No any type of biological software is present and the resulting actions are obtained by biological hardware. This biological hardware is the result of hundreds of synaptic connections among the different types of involved neurons.

Five are the basic neurons appearing in the retina structure. These neurons are photoreceptors, horizontal, bipolar, amacrine and ganglion cells. The first three types have a behavior similar to an analogical system, with responses with different levels according to the intensity of the impinging signal to the photoreceptors. On the contrary, amacrine and ganglion cells have responses similar to digital signals, with frequencies depending on the signals intensities. The most important processing place is related with amacrine cells. They interconnect bipolar neurons and different responses are obtained according to the existing synapses. These synapses have been created during the animal first years of life

Amacrines come in an astonishing variety of shapes and use an impressive number of synapses. There may be well over twenty different types. They all have in common, first, their location, with their cell bodies in the middle retinal layer and their processes in the synaptic zone between that layer and the ganglion cell layer; second, their connections, linking bipolar cells and retinal cells and forming an alternative route between them; and, finally, their lack of axons and a large ability of their dendrites to end on other cells.

Several different functions are performed with amacrine cells. Some types of amacrine seems to play a part in specific responses to moving objects found in retinas of frogs and rabbits; other types are interposed in the path linking ganglion cells to those bipolar cells that receive rod inputs. In some cases, they may be involved in the center-surround organization of ganglion-cell receptive fields.

Biuengineered and Bioinspired Systems III, edited by Paolo Arena. Angel Rodriguez-Vazquez. Gustavo Linan-Cembrano, Prac. of SPIE Vol. 6592, 65920H, (2007) - 0277-786X/07/\$18 - doi: $10.1117 / 12.722015$ 
Some of the more important work related with the retina has been achieved by John Dowling from 1960 to 1980. One of his books ${ }^{1}$ is a cornerstone in this field. A model of the retina appears there and it is given in Fig. 1. It shows the more elemental structure of the retina. with the five types of cells involved as well as a possible type of the obtained responses when some light impinges onto one of the two existing photoreceptors. The more interesting result appearing in the three ganglion cells corresponds to the appearing signals. Each one of the ganglion cells gives a different type of bursting signal. The first ganglion cell gives regular pulses before and after the light pulse and have a constant signal, when light appears. The third one offers a complementary behavior: no pulses when there is no light and a train of pulses when there is light. Finally, the middle one gives some few pulses at the beginning and at the end of the light pulse.



Fig. 1.- Dowling model In this work we will report a deeper analysis of the performance of some of the above mentioned more than forty types of existing amacrine cells.

We will analyze in this paper how this behavior may be emulated with a particular emphasis in the amacrine responses. The basis for our work will be our previously reported retinal model ${ }^{2}$. This model was based on the use of optically programmable logic cells, OPLCs, working as unit cells for the whole structure (fig. 2)

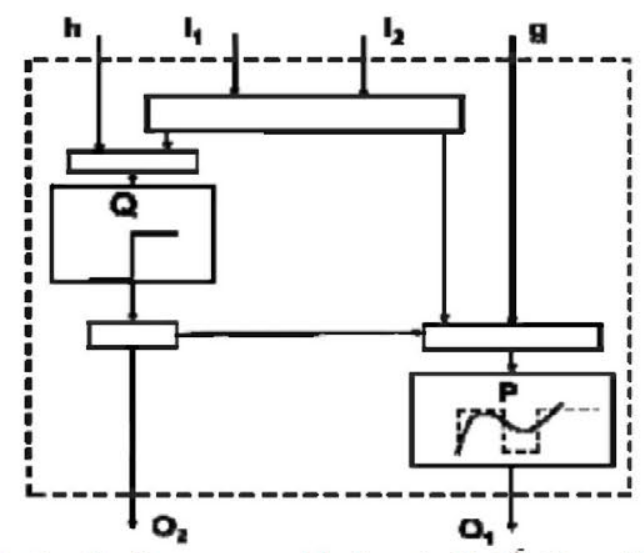

Fig. 2.- Basic structure of the Optically Programmable Logic Cell ${ }^{5}$. ( ) and $\mathrm{P}$ are non linear optical devices. 
A single basic cell. with the same structure in every one of the possible situations, has been employed. This cell has been extensively reported in several previous papers. It has been the basis for optical computing structures. Internal details appear in reference ${ }^{i-6}$. The same type of cells is employed as basis for the design of photoreceptors, horizontal, bipolar, amacrine and ganglion cells, being the sole difference the interconnections and the internal parameter values. This solution offers a very convenient way of working, because the differences in behaviour are obtained just with a different type of connection between different units.

\section{SOME BASIC DETAILS ABOUT THE OPLC BEHAVIOR}

As it has been pointed out before, just one single cell has been employed to construct the five retina layers. The basic scheme appears in Fig. 2. It is composed by two devices, $\mathrm{P}$ and $\mathrm{Q}$, with non-linear behaviour. Internal connections are represented in the figure. The outputs of each one of them correspond to the two final outputs, $\mathrm{O}_{1}$ and $\mathrm{O}_{2}$, of the cell. Four are the possible inputs to the circuit. Two of them are for input data, $\mathbf{I}_{\mathbf{1}} \mathrm{e} \mathbf{I}$, and the other two, $g$ and $\boldsymbol{h}$, may act as control signals. The way these four inputs are arranged inside the circuit, is also represented in Figure 1. Although a practical implementation we have carried out of the processing element has been based on an optoelectronic configuration, a computer simulation has been adopted for the present work. In a laboratory implementation, lines in Fig. 2 represent optical multimode fibres. The indicated blocks, placed in order to combine the corresponding signals, are conventional optical couplers. In this way, optical inputs arriving to the individual devices are multilevel signals. The characteristics of the nonlinear devices are also shown in Fig. 2. Device Q, corresponds to a thresholding or switching device, and device P is a multistate device, being the response of this non-linear optical device the one represented in Fig. 2 . This response is similar to the behaviour of a SEED device and it may be implemented by two semiconductor optical amplifiers, arranged in cascade, with a feedback from the second to the first one. Some details of this situation have been reported by us?

Because the cell works with optical signals, it allows an internal multilevel processing. In this way, input and output data signals are binary but the control is performed by multilevel signals. Table 1 lists the different programming combinations available from our Optical Programmable Logic Cell, OPLC. At least in one of the outputs, the eight Boolean functions - AND, OR, XOR. ON and the negative NAND, NOR, XNOR and OFF - can be obtained.

TABLE I.- Output functions of the optical-programmable logic circuit.

\begin{tabular}{|l||l|l|l|}
\hline \begin{tabular}{l} 
Q - Contro1 Signal \\
\hdashline P - Control Signal
\end{tabular} & $\begin{array}{l}\text { Q-Output: AND } \\
0-0.4\end{array}$ & $\begin{array}{c}\text { Q-Output: OR } \\
0.5-0.9\end{array}$ & $\begin{array}{c}\text { Q-Output: ON } \\
1.0-2.0\end{array}$ \\
\hline \hline $0-0.4$ & P - Output & $\begin{array}{c}\text { P - Output } \\
\text { X - Output }\end{array}$ \\
\hline 0.5 & NAND & XOR & NAND \\
\hline $0.6-0.9$ & NN & XNOR & NOR \\
\hline 1.0 & XNOR & XNOR & XNOR \\
\hline $1.1-1.4$ & XNOR & ON & AND \\
\hline 1.5 & AND & OR & OR \\
\hline $1.6-2.0$ & OR & OR & OR \\
\hline $2.0-2.5$ & ON & ON & ON \\
\hline
\end{tabular}

The importance of this behaviour, when applied to the simulation of the retina, it is large number of logic functions. As it is well known, according to the synapses implemented in any biological system, different responses are 
obtained and, all of them correspond to different logic functions obtained from the corresponding pulsed inputs. In the same way that similar neuron structures are able to perform very different sensory tasks, our single OPLC configuration, will be able to implement the different behaviours had in the retina layers.

\section{BASIC STRUCTURE OF THE SIMULATED RETINA}

The main blocks in our retina model appear in Figs. 3.a-b.

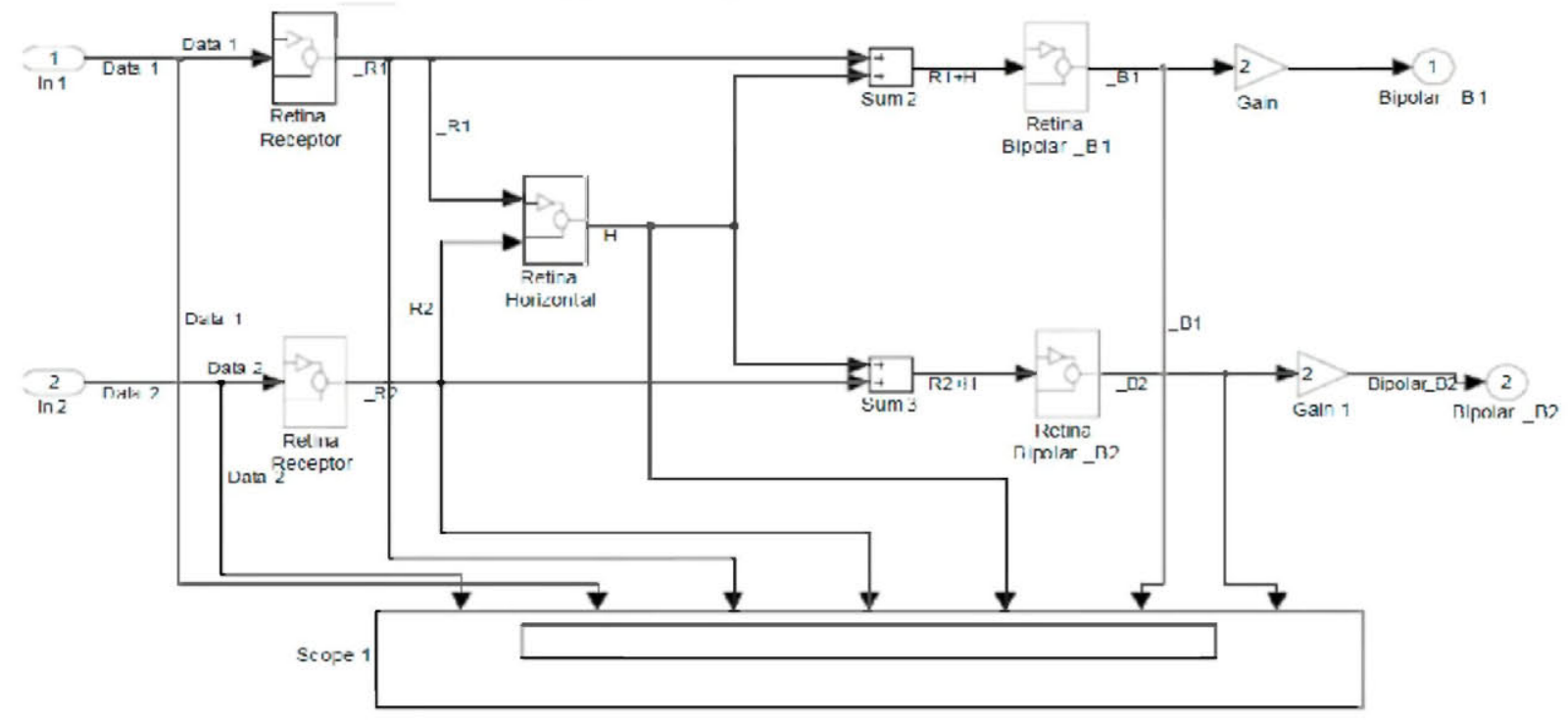

Fig. 3.a.- Configuration of the first three layers of the retina (Simulink simulation). Outputs go to the next two and final layers (Fig. 3.b)

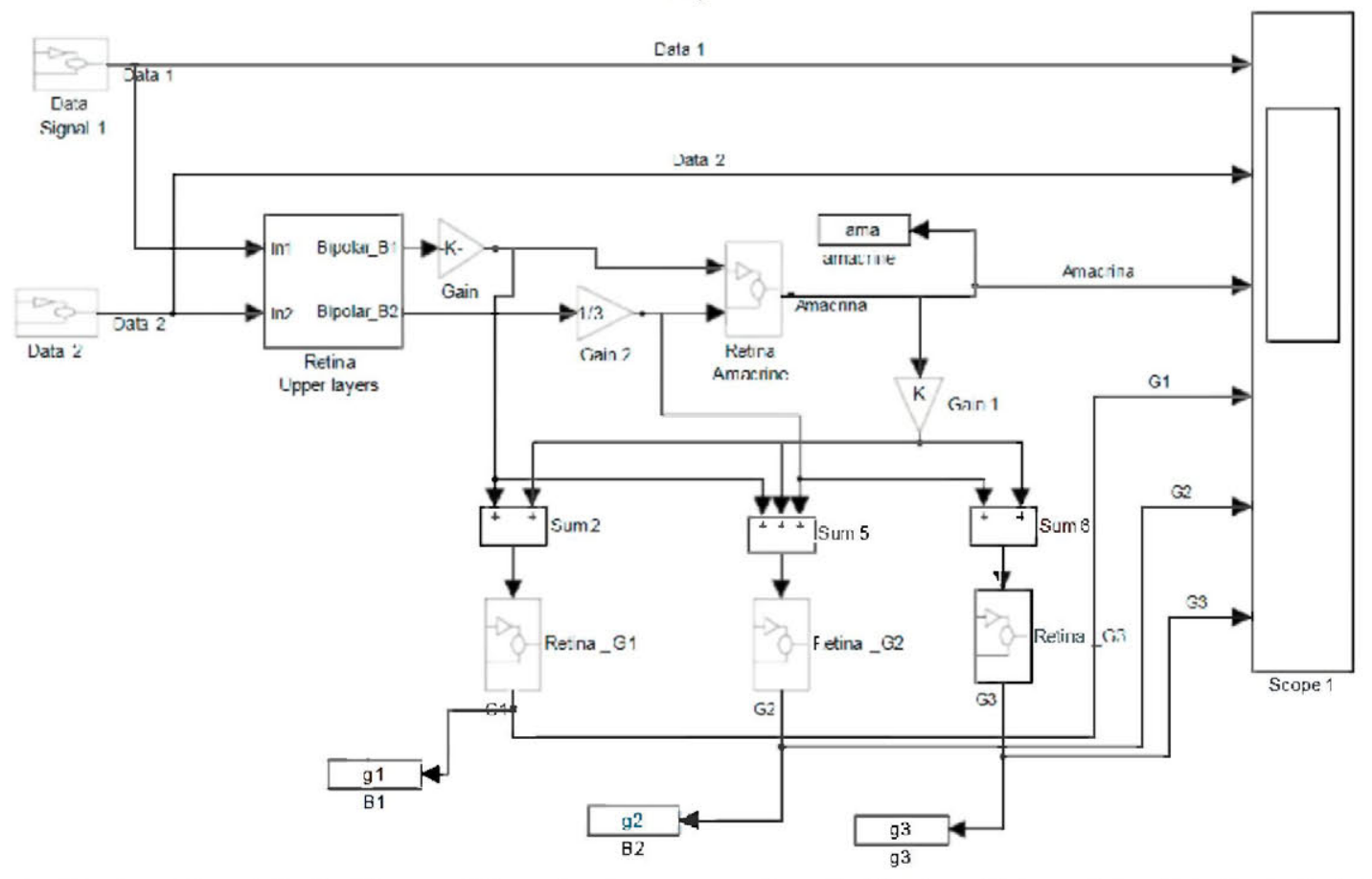

Fig. 3.b.- Configuration of the final layers of the retina. Inputs come from the bipolar cells layer (Simulink simulation). Block corresponding to Retina upper layers is indicated (Fig. 3.a) 
As it may be seen, the first three layers (Fig. 3.a) are arranged in a configuration following the Dowling model. No particular details are shown because they have reported previously ${ }^{2.4}$. Moreover we have considered in this part of the structure just the addition of input signals. On the contrary, the final layers, corresponding to the zone where amacrine and ganglion cells are located (Fig. 3.b), show some differences with respect to the Dowling model. The more important are the three amplifier blocks, two of them located before the amacrine cell and the third one, after the amacrine output. This configuration allows several possibilities for inputs to the ganglion cells. In the present case, inputs to the first ganglion are coming from one of the input amplifiers and from the amacrine output, The third ganglion cell get its inputs from the second input amplifier and from the amacrine output. Finally, the middle ganglion neuron gets three inputs, two of them coming from the two input amplifiers and the third one from the amacrine output. In this way, the information coming from upper layers is combined in three different ways in lower part of the retina. This fact gives several possibilities to process incoming data.

All the different retina neuron block, the receptor, horizontal, bipolar, amacrine and ganglion cells are the OPLC device. Different signals at the input and the corresponding Boolean function makes the behavior of each one OPLC block to emulate the real behavior of each retina neuron. The simulation model employed a fixed-step type with a discrete (no continuous states) solver and unconstrained periodic sample time.

Changes in the internal connections inside the amacrine cells may be added. This allows the possibility to obtain more complex outputs. This fact is due to the non linear behavior of the OPLC. Some possibilities have been shown by us previously, concerning this non linear behavior. As a matter of fact, in some case is possible to obtain a periodic output with a constant input and even a chaotic signal. This situation should be useful when some type of delusions should be studied in the visual system.

\section{ANALYSIS OF MOTION IN THE INPUT FIELD}

Two have been the analyzed situations with the above detailed model. The results, shown in Fig. 4, are for the case of two pulses of light impinging onto the photoreceptors, on different time intervals. Data 1 is the signals onto the photoreceptor 1 and Data 2, onto photoreceptor 2. Each one of them is indicated, in order to be differentiated, by different type of arrows. $\uparrow$ corresponds to beginning of Data 2 and $\downarrow$ to the final of the light pulse. Similar agreement corresponds to Data 1, with bold head arrows.

Data corresponds to input light pulses. In this first case, both have the same characteristics but they are separated in time. Responses in the photoreceptors are equivalent to the light pulses. It is necessary to point out that the rest state is taken at level 0 and the working state at level 1 . Responses in photoreceptors follow the pattern of the input light pulses but because in these cells the rest state corresponds to a level of 1 , when there is light the working condition goes to a 0 level. This situation is in a good agreement with the behavior of some types of cells.

The response in the horizontal cell is the addition of these two signals. Hence, with a rest state at a 1 level, signals at 0 levels appear when light goes onto both photoreceptors. Responses in bipolar neurons are similar to the responses of the corresponding photoreceptors.

Amacrine, the main objective of this work, offers a very different behavior. It has a response that, in some way, is similar to the obtained for the previous horizontal cell. It has a level of 1 , for the rest state and a 0 level when light goes onto the photoreceptors. But, as it can be seen in Fig 4, the transition from working level to rest level does not appear at the same time than in the horizontal cells. It appears a certain interval of time later. This delay has been generated in the amacrine cell according to the amplitude level of the incoming pulses - in our present case, always with the same level and, hence, the delay is always the same one. This is delay is fundamental for the final response of ganglion neurons.

The behavior of ganglion cells is very different depending on their position with respect to the input photoreceptors. All of them have a 0 level when they are on a rest state. The first one, the corresponding to the first photoreceptor, jumps to a level 1 when light is coming to its corresponding photoreceptor, going to the rest state after the above indicated delay. The main point is a new pulse appearing at the end of the pulse of light impinging onto the second photoreceptor. A similar situation, with the corresponding differences, is obtained in the third ganglion cell. The ganglion located in the middle is always on a level of value 1, but when there are light pulses in the input layer, the 
ganglion cell changes to a level 0 at a very short tune interval. This means that it is possible to know the end of both light pulses with this ganglion cell.
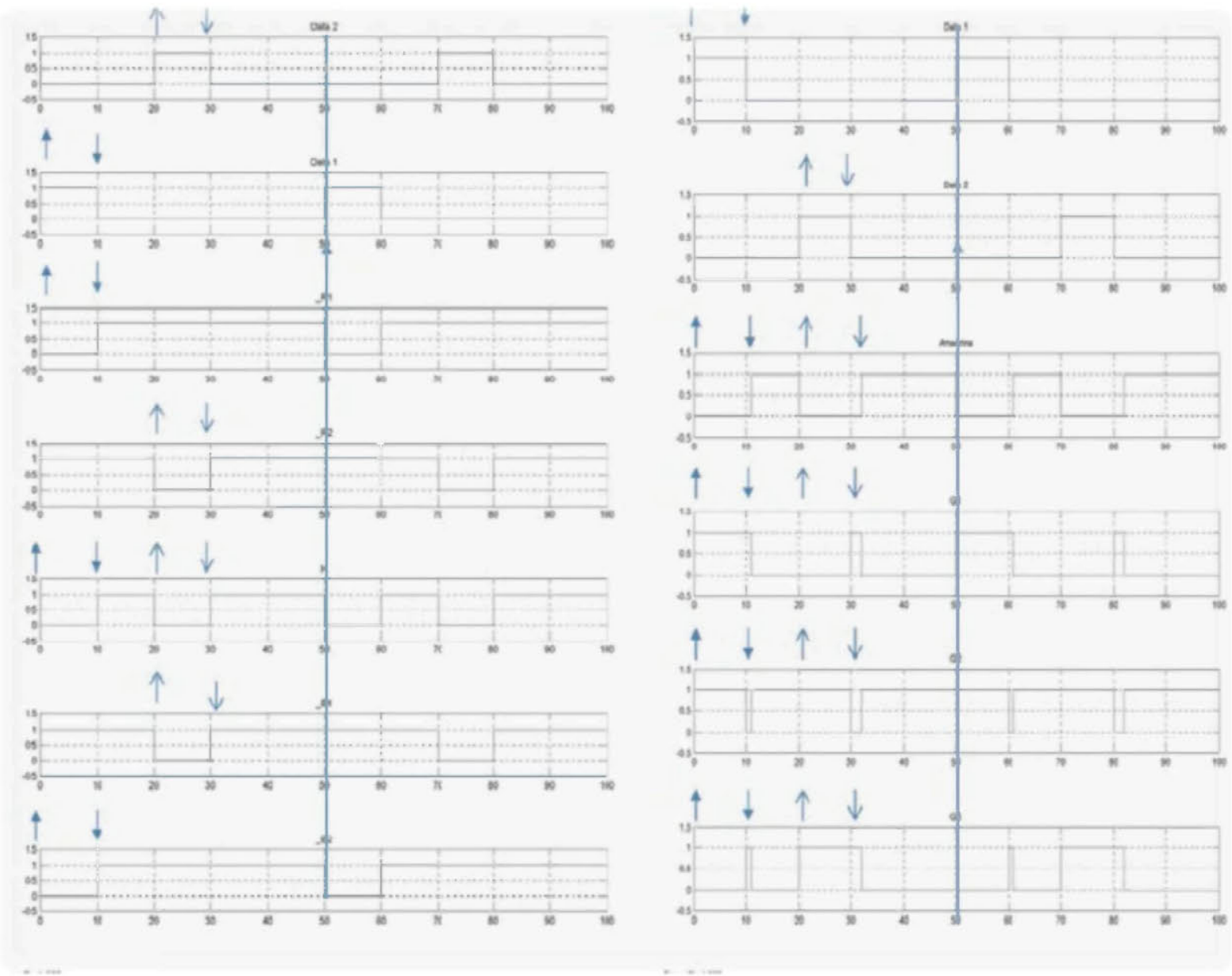

Fig. 4.- Outputs corresponding to the different cells in the retina model. Details are given in the text.

Fig. 5 shows a different situation. In this case, there is a certain overlapping in time between the incoming pulses to the photoreceptors. One of the more interesting new results appears analyzing the signals in the amacrine cell. In this case. this neuron is just in the rest state when there is no light pulse in any of the two receptors. Because the output signals from the ganglions are a result of the input and output signals from the amacrine, ganglions cells give now a very different result with respect to the previous one.

Ganglion cells change of state always that a change in level appears in any one of the retina previous layers.

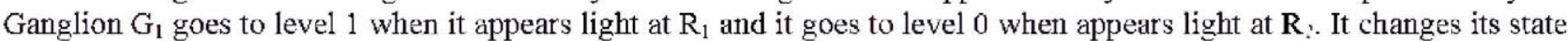
again when light disappears at $R$ !- with a very short pulse, returning to 0 . Ganglion cell $G_{3}$ is always at a 0 level and it changes only to level 1 in the time interval between the end of pulse impinging onto photoreceptor 1 and the end of pulse onto photoreceptor 2 - with the corresponding previously indicated delay. The signals in Ganglion 2 give information about both input lights pulses as well as overlapping times. As it may be seen in Fig. 5, any situation with a change in the input light conditions gives rise to a change in the level of the signal. In the reported case, there is a change from 1 to 0 or vice versa, at the beginning and at the end of input signals. 

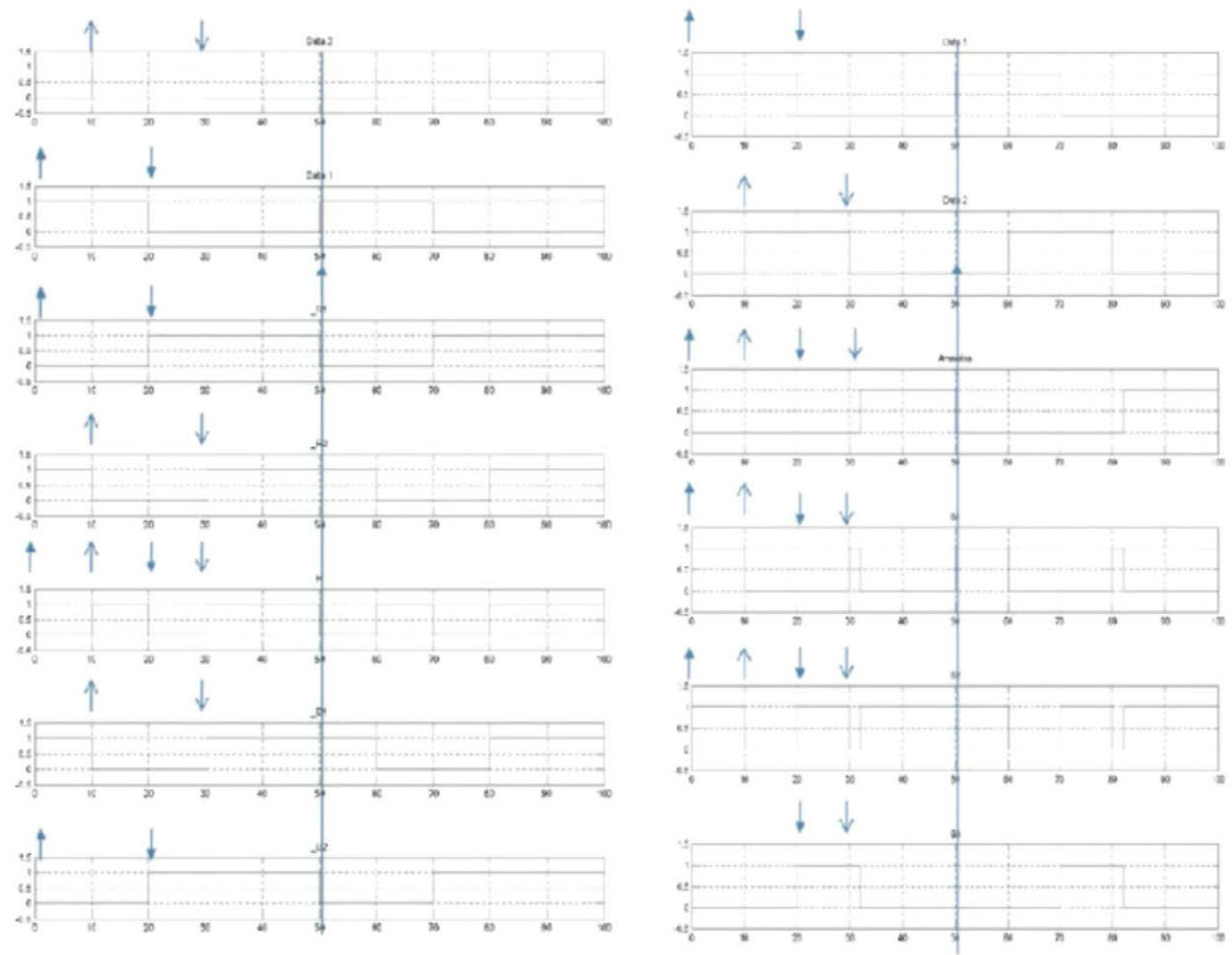

Fig. 5.- Outputs corresponding to the case when there is overlaping in the input light. Detals are given in the text.

\section{BEHAVIOR IN OTHER TYPES OF AMACRINES}

With the same Dowling model of figure 1 and simulation block of figure $3 \mathrm{a}$ and $3 \mathrm{~b}$. and just modifying the internal amacrine weights for the two inputs with a gain block, the signal generated by the ganglion cells are completely different, as it can be seen on figures 6 and 7 . On this figures, as an example, four types of amacrine respond are simulated. We can see that input data are the same as previously, and Amacrine output changes. Presently there is a classification of amacrine cells consisting of about 40 different morphological subtypes.

The information obtained from amacrine determines the signal send to the visual cortex by the retina via the ganglion neurons. Although we do not fully understand the neural code that the ganglion- cell axons send as trains of spikes into the brain, via the optic nerve, we are coming close to understanding how ensembles of ganglion cells respond differently to aspects of the visual scene and how fields of influence on particular ganglion cells are constructed. Much of the construction of the visual images does seem to take place in the retina itself, although the final perception of sight is indisputably done in the brain ${ }^{8}$. 

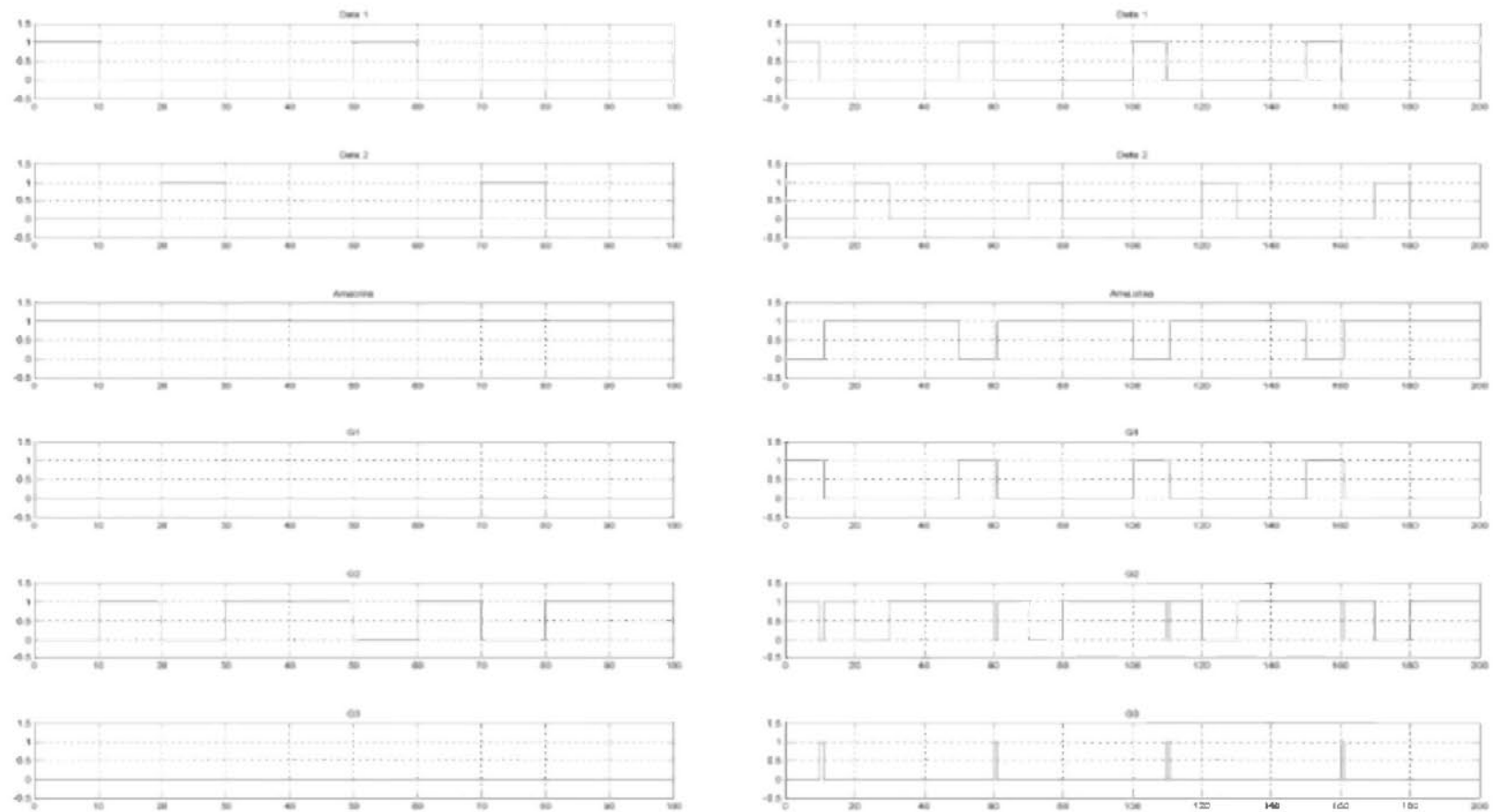

Fig. 6.- Outputs corresponding to the case when there is no overlaping in the input light. The only different between lefht and right correspond to the amacrine, the ganglion cells, G1, G2 and G3 are the same.
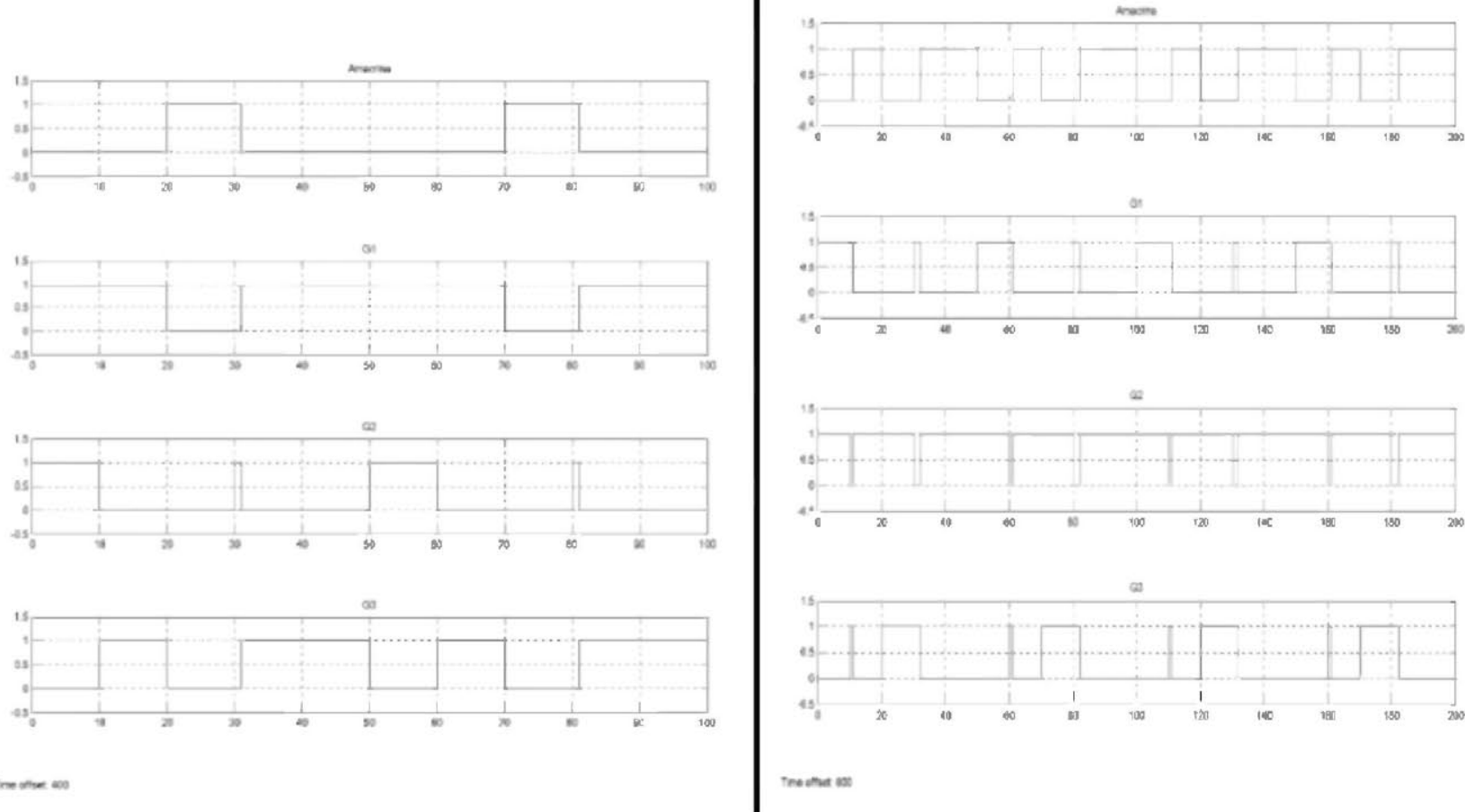

Fig. 7.- Outputs corresponding to the ease when there is no overlaping in the input light, see input light from figure 6 . The only different between leflit and right column and figure 6 correspond to the amacrine; the ganglion cells, G1, G2 and G3 are the same in the four cases represented. 


\section{CONCLUSIONS}

The reported analysis allows inferring under what type of conditions a certain light is crossing in the front of the two receptor of our model. The pulses indicated in the previous paragraph are a simulation of a light signal going from the right side to the left side. The real situation would be a point of light going from receptor 1 to receptor 2 , in the case of Fig. 4. The case in Fig. 5 should be of light with a certain area going also from receptor 1 to receptor 2 and with a certain receptors overlapping. Signals in any of the ganglion cells appearing in Fig. 5 are different from signals in Fig. 4.

These results, obtained with a very simplified model, can be easily generalized to a more complex structure. Following cases to be arialyzed should be with a larger number of photoreceptors and, as a consequence, larger number of cells in the following retina layers. But some of the main consequences are the reported in this work.

A further study, on what type of information is transmitted to the visual cortex from the retina depending on the amacrine processing. have to be done. From a single pulse of light no light. the retina model used predict that it is generating a lot more information than just anı input of motion.

\section{ACKNOWLEDGEMENTS}

This work was partly supported by: Ministerio de Educacion y Ciencia, project TIC 2003-04309 and TEC2006-13874-C02-02.

\section{REFERENCES}

1. J. Dowling. The Retina. An Approachable Part of the Brain. The Belknap Press of Harvard University Press, London, 1987.

2. J.A Martin-Pereda, Ana Gonzalez-Marcos "A Model of the Mammalian Visual System with Optical Logic Cells" Chapter 30- "Handbook of Neural Engineering", edited by Metin Akay and copublished by the IEEE Press and John Wiley \& Sorıs, Inic.. December 2006.

3. J.A. Martin-Pereda \& A. Gonzalez-Marcos, "A New Approach to Opttcal Fibre Sensing Techniques based on the Sensory Systems of the Living Bodies". In "Handbook of Optical Fiber Sensing Technology", edited by Jose Miguel Lopez-Higuera. Wiley, pp. 769-781. 2002.

4. A. Gonzalez-Marcos and J.A. Martín-Pereda, "A New Approach to a Model of the Mammalian Retina with Optically programmable Logic Cells". 1st. International IEEE EMBS Conference on Neural Engineering. Capri (Italia). $20-22$ de marzo, 2003. ISBN: 0-7803-7819-9

5. A. González-Marcos \& J.A. Martín-Pereda, "Digital Chaotic Output from an Optically-Processing Element". Optical Engineering, 35, pp. 525-535 (1996)

6. A. Gonzalez-Marcos \& J.A. Martín-Pereda, "Analysis of irregular behaviour on an optical computing logic cell". Optics \& Laser Technology, $32.457-466(2000)$

7. A. P. Gonzalez-Marcos, A. Hurtado, J.A. Martin-Pereda "Optical bistable devices as sensing elements" SPIE Vol. 5611, p. 63-70, Unmanned/Unattended Sensors and Sensor Networks; Edit.: Edward M. Carapezza. (2004)

8. Helga Kolb "How the retina Works" American Scientist, Volume 91, pp. $28-35$ (2003) 